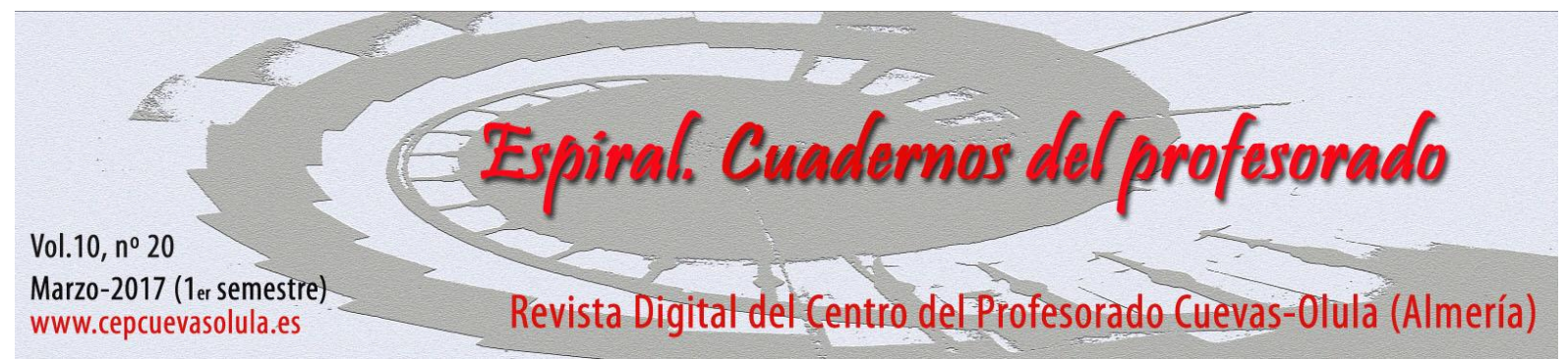

\title{
Historia a ritmo de rap. Una propuesta interdisciplinar para la enseñanza de las Ciencias Sociales y la Educación Artística
}

\section{Learning History to the Rhythm of Rap. An Interdisciplinary Approach to the Teaching of the Social Sciences and Arts Education}

\author{
Alejandro Egea Vivancos ${ }^{(1)}$, Laura Arias Ferrer ${ }^{(1)}$, \\ y María Esperanza Clares Clares ${ }^{(2)}$
}

\author{
(1) Área de Didáctica de las Ciencias Sociales, Facultad de Educación, Universidad de Murcia, España \\ ${ }^{(2)}$ Área de Música, Facultad de Educación, Universidad de Murcia, España
}

\begin{abstract}
RESUMEN: Este trabajo presenta y analiza una propuesta de innovación educativa llevada a cabo en Educación Secundaria cuyo objetivo es acercar al alumnado contenidos propios de la disciplina histórica a través de la música, en este caso concreto del rap. Gracias a sus versos, los alumnos pueden repasar los periodos de la historia mediante la lectura, escucha y análisis de la canción "Mil vidas", del artista español Nach. Tras esta actividad, se solicita a los alumnos un ejercicio creativo por el que escriben y recitan pequeñas estrofas compuestas por ellos mismos. Este esfuerzo narrativo no solo permite repasar y reflexionar sobre los contenidos históricos aprendidos durante el curso, sino que también favorece la introducción de rimas y ritmos. La actividad es fácilmente adaptable a diferentes cursos y consigue aumentar la motivación del grupo hacia la materia además de posibilitar la evaluación de algunos contenidos aprendidos, valorando el significado dado por el alumnado a determinados acontecimientos de la historia. Además, sirve de ejemplo de actividades interdisciplinares. En este caso, música e historia se conjugan para captar la atención del alumnado y contribuir a una mejora del proceso de enseñanza-aprendizaje.
\end{abstract}

Palabras clave: Enseñanza de la Historia; Música; Formación del Profesorado; Educación Secundaria; TIC.

\begin{abstract}
This work shows and analyzes an educational proposal conducted in Secondary Education, whose main aim is to bring the students closer to history by using music as a teaching tool. In this specific case, students go over periods of history through the rhythm of a rap. Students read, listen and analyze the verses of the song "Mil Vidas", of the Spanish singer Nach. After this first task, students are asked to develop a creative exercise where they write and sing their own strophes. This narrative effort not only allows them to go over and think about the historical contents learnt during the course, but also to introduce students to rhyme and rhythm. The activity is easily adaptable to different levels, gets students to increase their motivation to the subject and allows teachers to assess some historical contents by analyzing the meaning given by the students to some historical events and agents. Besides, it serves as an example of interdisciplinary activities. In this case, music and history are combined in order to catch the attention and interest of the students and to improve the teaching and learning process.
\end{abstract}

Key words: History Education; Music Education; Teacher training; Secondary Education; ITC.

Egea Vivancos, A., Arias Ferrer, L., y Clares Clares, M. E. (2017). Historia a ritmo de rap. Una propuesta interdisciplinar para la enseñanza de las Ciencias Sociales y la Educación Artística. Espiral. Cuadernos del Profesorado, 10(20), 51-57. Disponible en: http://espiral.cepcuevasolula.es/

Fecha de recepción: 14/09/2016

Fecha de aceptación: 15/01/2017
Enviar correspondencia a: alexegea@um.es 
Toda una vida es poco para un hombre, la Tierra me entregó un cuando y un donde atrapado ahora en esta era insomne, quiero vivir más vidas que la que me corresponde...

Nach, Mil Vidas (Un día en Suburbia, 2008)

\section{Introducción}

Con estos versos comienza la línea del tiempo creada y rapeada por Nach. Escucharla casi por casualidad sirvió para poner en funcionamiento la máquina de los recursos. Y es que un profesor debe buscar y crear recursos y estrategias de donde sea para procurar conseguir metas que se nos presentan cuan piedras filosofales del proceso de enseñanza-aprendizaje: el aprendizaje significativo y, a través de él, conseguir un recuerdo de lo aprendido de largo recorrido. Y es que, por desgracia, al menos en lo referente a los contenidos propios de Ciencias Sociales, se ha detectado un aprendizaje bastante efímero entre el alumnado que inicia sus estudios universitarios (Navarro-Medina \& Alba-Fernández, 2014). Es realmente pasmosa la velocidad con la que se olvidan contenidos que nos consta, por propia experiencia, que han sido repetidos en varias ocasiones a lo largo de la Educación Primaria y Secundaria.

Ante este recurso, nos resultaba más que interesante indagar sobre otras maneras de acercar al estudiante a la historia. En este sentido, lo audiovisual pensamos que tiene mucho que decir. La música nos puede servir para contextualizar una época, crear un ambiente de autenticidad en el aula o utilizarla como fuente primaria (Murphy, 2011), pero también, como es el caso, para acercar los contenidos de una manera cercana al alumnado del siglo XXI. Sin embargo, no es lo mismo contextualizar la época del Barroco escuchando a Bach, estrategia histórica y didácticamente impecable, que mostrar a nuestro alumnado contenidos históricos mediante estilos más cercanos a ellos.

No cabe duda, como defendía Gardner en su teoría de las inteligencias múltiples (2011), que la inteligencia musical es una de las diferentes maneras que poseen los estudiantes para aprender. Este tipo de inteligencia pone en juego habilidades como la composición, la actuación y la apreciación de patrones musicales. En este sentido, establecer la posibilidad de que en el aula el alumnado, de cualquier edad, pueda construir sus propias composiciones siguiendo un tipo de poesía urbana es una actividad eminentemente creativa. En palabras de Turner (2008, p. 13), "children can dance, move, and add sound effects to a rap that they or the teacher have created. Rapping can provide a good vehicle for carrying the content of a lesson". Así mismo, si la historia es vista en ocasiones por el alumnado como algo aburrido y condenado a la memorización, la música y el análisis que se puede realizar sobre ella pueden aportar a los profesores la oportunidad de motivar a sus estudiantes en una mayor medida, como pocas otras fuentes pueden conseguir (Pellegrino \& Lee, 2012). La idoneidad de este recurso para el proceso de enseñanza-aprendizaje aparece avalada por diversos trabajos en los que se analiza e incorpora el hip-hop y la cultura asociada a la misma, en las aulas educativas (Alonso \& Barba, 2013; Herrera \& Cremades, 2010; Morgade, Poveda \& Verdesoto, 2016; Rodríguez \& Iglesias, 2014). Además, si en la experiencia de aula se integran las nuevas tecnologías, el proceso de enseñanza-aprendizaje será mucho más motivador y enriquecedor (Giráldez, 2015).

\section{Origen de la Experiencia}

La actividad que se presenta ha sido desarrollada y probada en el IES El Bohío de Cartagena, concretamente entre el alumnado de $2 .^{\circ} \mathrm{ESO}$ durante tres cursos académicos. El centro está ubicado en la periferia de la ciudad y se nutre de barriadas y pueblos próximos. Dicho alumnado posee una procedencia muy heterogénea al venir de ámbitos tanto urbanos como rurales. La motivación hacia los estudios en los primeros cursos de la ESO era algo escasa en determinados grupos. En algunas clases, aquellas más disruptivas, la predisposición hacia el estudio era prácticamente inexistente. Por este motivo, se planteó en un momento determinado una manera novedosa de trabajar y repasar ciertos contenidos de Historia en un grupo muy reducido (de tan sólo 13 alumnos) que sin embargo poseía un alto nivel de disrupción y desinterés hacia la materia. 


\section{Objetivos}

La actividad en concreto fue diseñada para repasar los contenidos de Edad Media y Edad Moderna impartidos en $2 .^{\circ}$ ESO. Entre los objetivos que nos planteamos a la hora de realizar esta actividad cabe destacar los siguientes:

- Repasar algunos de los contenidos básicos vistos durante el curso.

- Fortalecer las destrezas relativas al uso y comprensión de la cronología.

- Trabajar la expresión literaria a través de las rimas.

- Fomentar el trabajo en grupo.

- Valorar la creación artística.

- Acercar la historia al alumnado de una manera más amena.

Así mismo, muchos son los contenidos que se desarrollaron con la actividad. La adaptación de la rima a desarrollar permite variar los contenidos meramente conceptuales (en nuestro caso Edad Media y Edad Moderna), pero la propia dinámica posee unos contenidos fijos. Citamos los más obvios:

- Comprensión de términos históricos nuevos y repaso de otros.

- Localización en el tiempo y en el espacio de periodos y acontecimientos históricos.

- Obtención de información a la hora de realizar las rimas, basándose en el libro de texto y sus notas de clase.

- Medidas de tiempo: milenio, siglo, año (Figura 1).

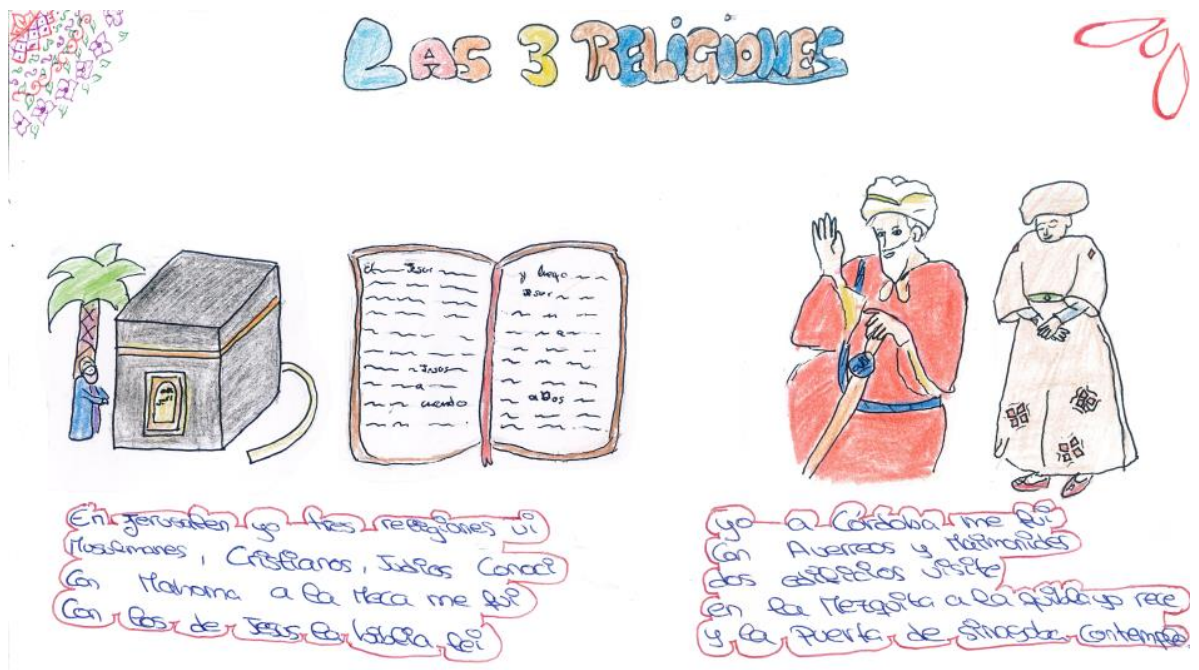

Figura 1. Ejemplo de composición a modo de rap de alumnado de $2 .^{\circ}$ ESO.

Por último, una actividad como esta sirve para desarrollar varias competencias básicas. Por ejemplo, nos resultaba elemental el fomento de la competencia lingüística. Por esta razón, los alumnos leyeron y trabajaron la canción, así como compusieron sus propias estrofas cuidando de la rima y el ritmo. El alumno, de manera autónoma, buscaba en los contenidos dados en sus notas y libro de texto, elegía y escogía la información, para construir sus pequeñas narrativas, trabajando así sin darse cuenta la competencia de aprender a aprender.

\section{Metodología}

Nuestra propuesta se basa en el tema "Mil vidas", incluida en el quinto álbum de Nach, Un día en Suburbia (2008). Ignacio Fornés, más conocido como Nach, es un artista y Master of Ceremonies [MC] español, básico para los amantes del rap. De hecho, entre los alumnos es bastante conocido. Fácilmente localizable en la web, la canción en sí misma es un eje cronológico rapeado en el que el 
autor selecciona aquellos periodos históricos en los que le hubiera gustado vivir, aquellos personajes históricos que le hubiera gustado ser. Con origen en la Prehistoria, pasando por el Antiguo Egipto, la Grecia Clásica, el Renacimiento o la Revolución Rusa, por ejemplo, Nach culmina su línea del tiempo con referencias recientes del siglo XX y personajes más cercanos a la cultura o el movimiento artístico hip hop como es el breaker Crazy Legs o la activista social Rosa Parks (Tabla 1).

$\mathrm{Al}$ alumnado se les hace llegar impresa una copia de la letra, con los versos numerados en su margen izquierdo. Esta numeración les servirá a posteriori para cumplimentar las actividades asociadas a la fase 2. Así mismo, es necesario una base de rap sin letra, sólo música, que servirá para desarrollar la fase 3.

Como hemos dicho, los acontecimientos de "Mil Vidas" han sido escogidos por el artista en base a sus preferencias por lo que, históricamente hablando, esta línea del tiempo queda descompensada ya que más del $50 \%$ de la letra se centra en el siglo XX. Por esa razón, las actividades propuestas al alumnado, además de servir para repasar los periodos que aparecen, sirven para intentar cubrir esos vacíos históricos.

La estrategia diseñada se puede dividir en cuatro fases. Por un lado, aquellas que están destinadas a repasar los conceptos históricos mencionados en la canción, así como su cronología y, por el otro, otras que instan al alumnado a recordar otros periodos de una manera creativa (Figura 2). Por último, la actividad finaliza con una puesta en común de todos los textos creados en la fase dos y una recapitulación por escrito.

La actividad está diseñada para 1 hora y media, máximo 2 horas, distribuidas en diferentes sesiones. Si el grupo es pequeño puede perfectamente organizarse a través de trabajo individual. Sin embargo, se enriquece mucho más si se forman pequeños grupos de tres a cinco personas.

\section{Adaptabilidad de la Experiencia}

La experiencia que describimos es fácilmente adaptable a otros niveles, incluido el alumnado universitario, con pequeños ajustes (Egea \& Arias, 2015). Durante varios cursos ha sido empleado este recurso con el mismo fin en el Grado de Educación Primaria, de cara a repasar contenidos y mostrar recursos de innovación a los futuros docentes.

Además, dado que en el rap se describen contextos pluriculturales muy diversos, la experiencia también es idónea para trabajar la interculturalidad, un proceso de comunicación e interacción entre culturas que la música favorece especialmente (Bernabé, 2012).

\begin{tabular}{lll}
\multicolumn{3}{c}{ Así mismo, el interés que la } \\
música suscita entre nuestros \\
alumnos, unido a su extraordinaria
\end{tabular}

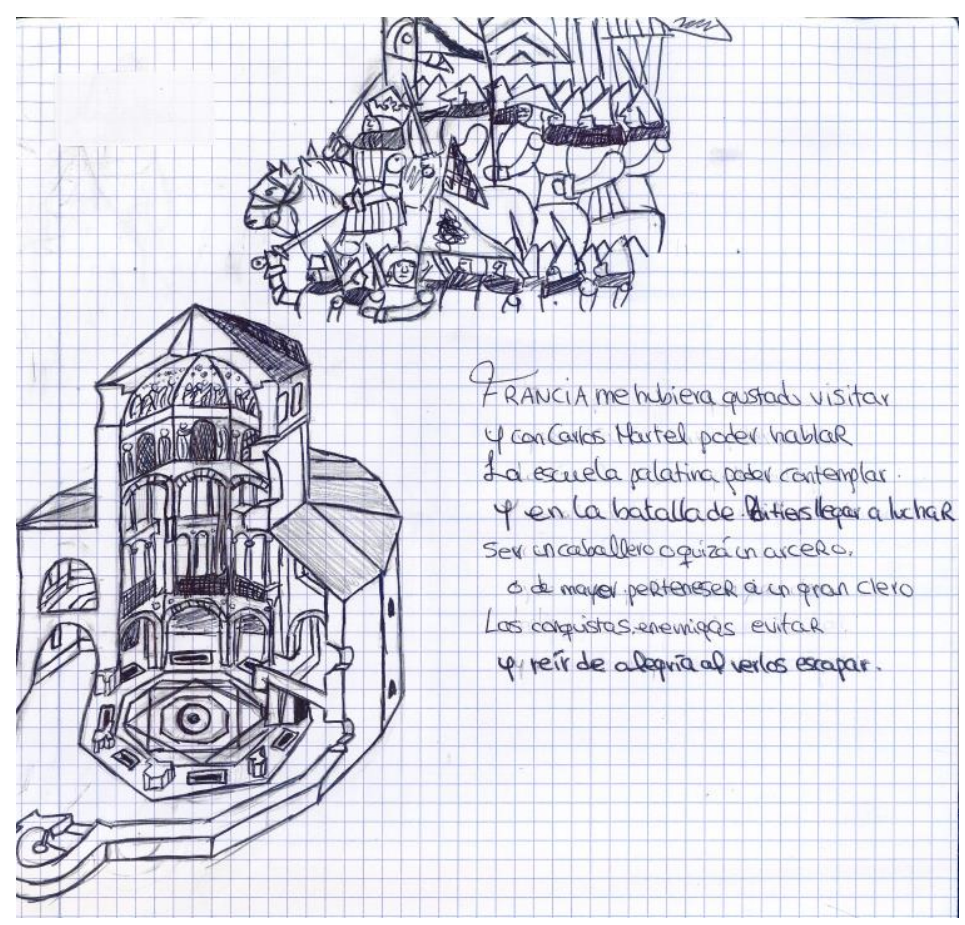

Figura 2. Ejemplo de composición a modo de rap de alumnos

de $2 .^{\circ} \mathrm{ESO}$.

facilidad para manejar dispositivos táctiles y móviles, nos inspira otras adaptaciones de la actividad (Tabla 2). Por ejemplo, una fase complementaria consistiría en crear una base original para rapear las rimas creadas, empleando herramientas y recursos digitales gratuitos y disponibles en Internet. El objetivo es que nuestro alumnado experimente con lo sonoro en la elaboración de producciones multimedia propias. 


\section{Tabla 1}

Fases y tareas que comprende la actividad

FASE 1 1. Escuchamos la canción y subrayamos:

a. Las palabras que no entendemos para luego preguntarlas.

b. Las palabras relacionadas con la Historia que conozcamos.

2. ¿En qué líneas habla de la Prehistoria? ¿Cómo lo sabes?

3. ¿En qué líneas habla de la Edad Antigua? ¿Cómo lo sabes?

4. ¿En qué líneas habla de la Edad Media y la Edad Moderna?

5. ¿A partir de qué líneas habla de la Edad Contemporánea? ¿Cómo lo sabes?

6. Si tuvieras que hablar de Al-Andalus o de Carlomagno, a partir de qué línea lo incluirías para que no se perdiera el orden de la historia?

7. ¿A qué línea crees que se corresponden los siguientes años? 1500, 450 a.C., 2700 a.C., 100000 a.C., 1969.

FASE 2 En la canción, la historia medieval (o la época romana) casi no está desarrollada. En grupo, y sin ayuda, inventamos y escribimos DOS estrofas (ocho versos) sobre aquellos episodios de la Edad Media (o la época romana) que creéis que deben ser destacados. Hay que procurar cuidar la rima.

Esta parte se puede cambiar para que cada grupo realice unas estrofas sobre un periodo a elegir o sobre aquel otro periodo que el docente quiera trabajar con más profundidad.

FASE 3 Puesta en común de las rimas creadas. A la hora de leerlas se reproduce una base de música rap sin letra para que los diferentes grupos intenten adaptar sus rimas a la música.

FASE 4 Con el tiempo sobrante se crean pequeños carteles que incluyen sus rimas y dibujos que ilustren las mismas.

La propuesta se llevaría a cabo en el Aula de Música; requeriría de, al menos, dos sesiones; y puede realizarse individualmente o por grupos. Existe un buen número de aplicaciones para crear música en móviles y tabletas. Para la actividad se ha elegido GrooveMixer, un generador de ritmos sencillo e intuitivo en el que el usuario compone por celdas, es decir, asignando un sonido a cada pista y distribuyendo por la cuadrícula los pulsos necesarios para generar patrones rítmicos y melódicos (Figura 3). Al patrón creado (en secciones de 16 tiempos) se le pueden aplicar, posteriormente, múltiples efectos. Además, la aplicación permite compartir las creaciones en Soundcloud y guardarlas como pistas de audio en los formatos habituales de MP3 o WAV, entre otros.

Dado que el interés de esta actividad radica en la experimentación que se produce durante el proceso creativo, se recomienda probar distintos tempos (entre 90 y $100 \mathrm{Bpm}$ ) y la combinación de sonidos (máximo ocho) de entre los más de 250 disponibles en la biblioteca de la aplicación.

\section{Tabla 2}

Adaptación de las Fases 3 y 4 incorporando las TIC

FASE 3 Puesta en común de las rimas creadas. Se puede llevar a cabo de dos formas:

- Grabarla previamente, utilizando, por ejemplo, http://audacity.sourceforge.net

- Rapearla en clase sobre la base instrumental de "Mil Vidas" de Nach, que podemos encontrar en https://soundcloud.com/mcjehc/nach-mil-vidasinstrumental. Con el fin de adaptar el rap al ritmo de la base, se recomienda empezar trabajando el pulso. Así su interiorización facilitará en gran medida el trabajo de interpretación.

FASE 4 Con el tiempo sobrante pueden crearse graffitis que ilustren las rimas creadas, utilizando alguna de las aplicaciones para móviles y tabletas disponibles gratuitamente en la Red. Graffiticreator (http://www.graffiticreator.net/), por ejemplo, permite hacer pequeñas ilustraciones y logotipos on line. 


\section{Evaluación}

La actividad fue diseñada y desarrollada con gran éxito en varios grupos de $2{ }^{\circ}$ ESO durante tres cursos académicos. En este caso, sirvió para repasar con los grupos los contenidos históricos trabajados durante este nivel. En el caso concreto de nuestro alumnado la motivación durante todo el tiempo que duró la actividad fue muy alta independientemente de las características del grupo (Tabla $3)$.

\section{Tabla 3}

Ejemplos de composiciones de los alumnos

\begin{tabular}{cc}
\hline Como Carlomagno quiero ser & En Jerusalén yo tres religiones vi, \\
Capilla palatina de Aquisgrán ver. & musulmanes, cristianos y judios conocí. \\
Quisiera ver las luchas por el poder, & Con Mahoma a la Meca me fui, \\
y me gustaría mi Imperio verlo crecer. & con los de Jesús la Biblia leí. \\
Como Abderramán I quisiera ser & Yo a Córdoba me fui \\
De sus luchas quisiera aprender & con Averroes y Maimónides dos edificios visité. \\
para emir llegar a ser & En la Mezquita a la quibla yo recé \\
ver la Mezquita de Córdoba crecer. & y la puerta de la Sinagoga contemplé. \\
\hline
\end{tabular}

Además, se trataba de una tarea que se adaptaba perfectamente a las distintas capacidades del alumnado, favoreciendo así la atención a la diversidad. Una prueba más de este compromiso con la actividad es que los carteles creados con sus letras para decorar el aula fueron celosamente respetados incluso en los grupos más disruptivos (Figura 4).

La aparición de un elemento novedoso en una clase de Ciencias Sociales siempre sirve para captar la atención del alumno. En este caso, la presencia de música urbana, de un artista que muchos de ellos conocen y que incluso han escuchado en varias ocasiones, les permite un acercamiento a la historia mucho más ameno y que nuestro alumnado acabe "historiando" a ritmo de rap o rapeando al ritmo de la historia casi sin darse cuenta.

Además, cabe recordar que experiencias como esta facilitan una socialización a través de la música, ya que es a través de ella sobre la que en parte construyen su propia identidad (Morgade, Poveda, \& Verdesoto, 2016). Junto a esto, el análisis de las respuestas del alumnado al analizar los versos y, lo que es más importante, el resultado obtenido tras el ejercicio creativo propuesto, permite evaluar la comprensión del alumnado respecto a determinados contenidos históricos, así como el significado que los estudiantes dan a determinados acontecimientos del pasado. La selección o no de determinados hechos y personajes, las descripción y/o acción que los acompaña, y el lugar que ocupan ellos mismos (los autores del verso) en la narración, son claves en este análisis (Egea \& Arias, 2015). Sus narrativas nos permiten determinar qué aspectos de la historia son considerados relevantes por el

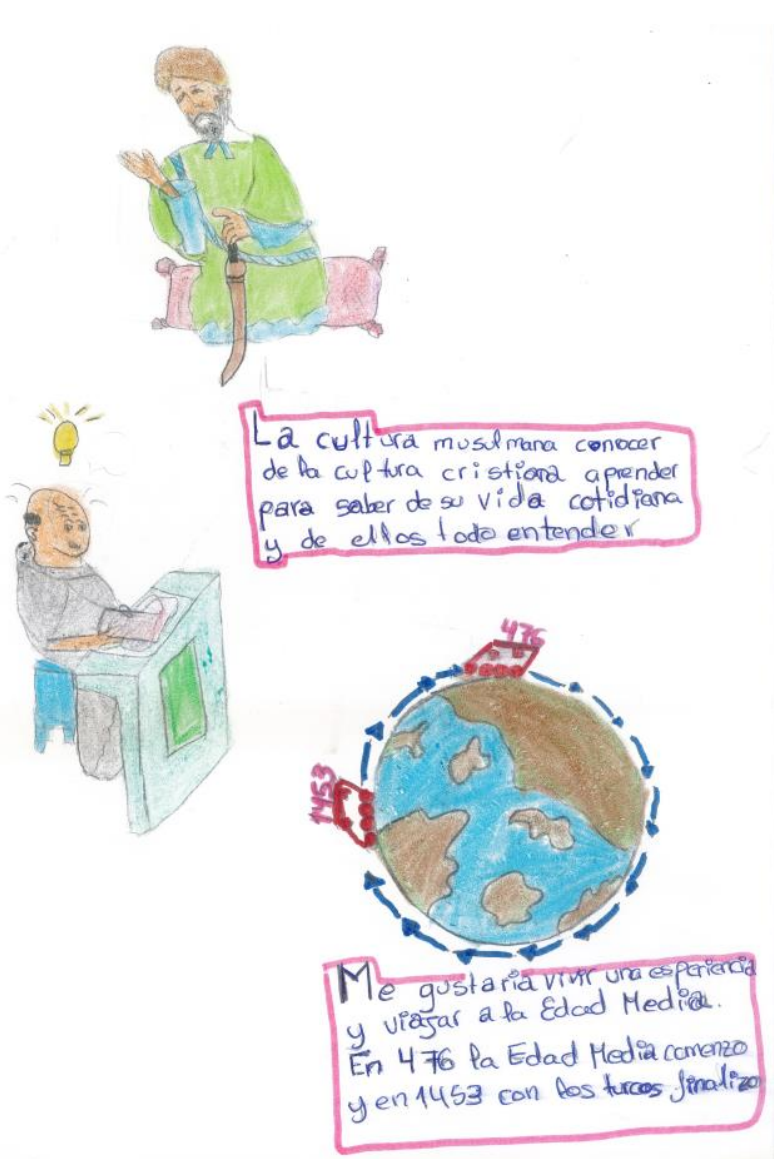

Figura 3. Ejemplo de composición a modo de rap de alumnos de $2 .^{\circ} \mathrm{ESO}$. 
alumnado, qué actores o agentes la protagonizan así como cuál su propia consideración respecto al propio devenir histórico.

Los buenos resultados obtenidos en el aula nos animan a aconsejar la realización de esta práctica en otros centros, así como en otros cursos de la Educación Primaria (fácilmente adaptable a $5 .^{\circ}$ o $6 .^{\circ}$ de Educación Primaria) y, por supuesto, en cualquier curso de Educación Secundaria con pequeñas modificaciones.

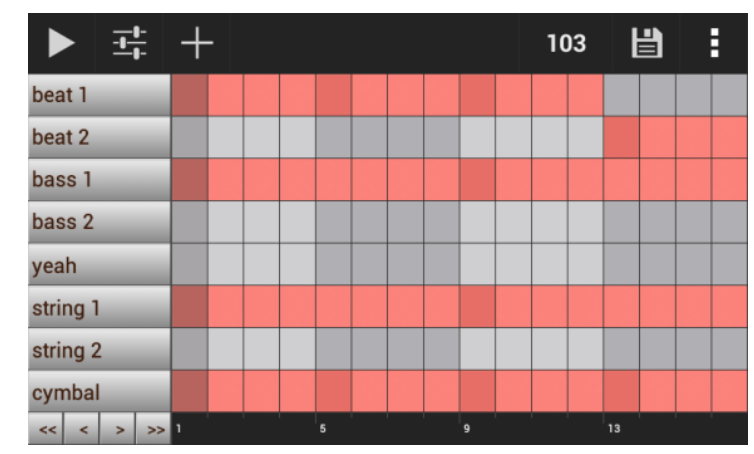

Figura 4. Cuadrícula de composición de ritmos de la aplicación GrooveMixer.

\section{Referencias}

Alonso, H., \& Barba, J. J. (2013). El grafiti en educación de calle para el fomento de la autoestima, las relaciones sociales y la promoción social: el caso de Espacio Mestizo. Revista Electrónica Interuniversitaria de Formación del Profesorado, 16 (3), 49-60.

Bernabé, M. (2012). La comunicación intercultural a través de la música. Espiral. Cuadernos del Profesorado, 5(10), 87-97. Disponible en: www.cepcuevasolula.es/espiral.

Egea, A., \& Arias, L. (2015). Russell Crowe el gladiador, Colón el descubridor y otros protagonistas de la Historia. Buceando en las narrativas históricas de jóvenes universitarios españoles». CLIO. History and History teaching, 41. Disponible en: http://clio.rediris.es.

Gardner, H. (2011). Frames of Mind: The Theory of Multiple Intelligences. New York: Basic Books.

Giráldez, A. (coord.) (2015). De los ordenadores a los dispositivos móviles. Propuestas de creación musical y audiovisual. Barcelona: Graó.

Herrera, L., \& Cremades, R. (2010). Preferencias musicales de los estudiantes de Educación Secundaria Obligatoria: influencia de la educación formal e informal. Cultura y Educación: Revista de teoría, investigación y práctica, 22 (1), 37-51.

Morgade, M., Poveda, D., \& Verdesoto, A. (2016). El barrio entra en la escuela a ritmo de hip hop. Eufonía. Didáctica de la Música, 68, 7-14.

Murphy, J. (2011). Más de 100 ideas para enseñar historia: Primaria y Secundaria. Barcelona: Graó.

Navarro-Medina, E., \& Alba-Fernández, N. (2014). La formación ciudadana a partir de la enseñanza de la Historia de España: cuatro situaciones para reflexionar. En N. Martínez-Valcárcel (Ed.), La historia de España en los recuerdos escolares: análisis, interpretación y poder de cambio de los testimonios de profesores y alumnos (pp. 267-286). Valencia: Nau Llibres.

Pellegrino, A. M., \& Lee, C. D. (2012). Let the music play!: harnessing the power of music for history and social studies classrooms. Charlotte, N.C.: Information Age Pub.

Rodríguez, A., \& Iglesias, L. (2014). La «cultura hip hop»: revisión de sus posibilidades como herramienta educativa. Teoría de la educación, 26 (2), 163-182.

Turner, T. (2008). The Bear is Still Singing: Creating Lyrics with Social Studies. Social Studies and the Young Learner, 21(1), 11-14.

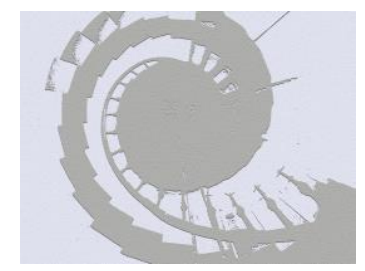

